\title{
Mobile robot navigation and obstacle avoidance techniques: A review
}

\begin{abstract}
Mobile robot is an autonomous agent capable of navigating intelligently anywhere using sensor-actuator control techniques. The applications of the autonomous mobile robot in many fields such as industry, space, defence and transportation, and other social sectors are growing day by day. The mobile robot performs many tasks such as rescue operation, patrolling, disaster relief, planetary exploration, and material handling, etc. Therefore, an intelligent mobile robot is required that could travel autonomously in various static and dynamic environments. Several techniques have been applied by the various researchers for mobile robot navigation and obstacle avoidance. The present article focuses on the study of the intelligent navigation techniques, which are capable of navigating a mobile robot autonomously in static as well as dynamic environments.
\end{abstract}

Volume 2 Issue 3 - 2017

\author{
Anish Pandey,' Shalini Pandey,' Parhi DR² \\ 'Oriental Group of Institutes, India \\ ${ }^{2}$ National Institute of Technology, India
}

Correspondence: Anish Pandey, Oriental Group of Institutes, India, Email anish06353@gmail.com

Received: January 07, 2017 | Published: May 23, 2017

Keywords: mobile robot, sensor, actuator, navigation, obstacle avoidance

Abbreviations: GA, genetic algorithm; NN, neural networks; PSO, particle swarm optimization; PWM, pulse width modulation; RNW-PSO, random inertia weight particle swarm optimization; ANN, artificial neural network; FL, fuzzy logic; Gas, Genetic Algorithms; MOGA, multiple objective genetic algorithm; SAA, simulated annealing algorithm; ACO, ant colony optimization; MPSO, modified particle swarm optimization; PSO, particle swarm optimization; RAOFC, reinforcement ant optimized fuzzy controller.

\section{Introduction}

This article introduces the literature survey of the various techniques used for mobile robot navigation. Navigation and obstacle avoidance are one of the fundamental problems in mobile robotics, which are being solved by the various researchers in the past two decades. The aim of navigation is to search an optimal or suboptimal path from the start point to the goal point with obstacle avoidance competence. Basically, the mobile robot navigation has been done by the Deterministic algorithm and Nondeterministic (Stochastic) algorithm. Nowadays, the hybridization of both the algorithms called as an Evolutionary algorithm is being used to solve the mobile robot navigation problem. Figure 1 shows the general classification of the Deterministic algorithm, Nondeterministic (Stochastic) algorithm, and Evolutionary algorithm, which are implemented for mobile robot navigation by various authors.

Navigation is an essential task in the field of mobile robotics, which can be classified into two types: global navigation and local navigation. ${ }^{1}$ In the global navigation, the prior knowledge of the environment should be available. Many methods have been developed for global navigation, i.e. Voronoi graph, ${ }^{2,3}$ Artificial potential field method, ${ }^{4,5}$ Dijkstra algorithm, ${ }^{6}$ Visibility graph, ${ }^{7}$ Grids,${ }^{8}$ and Cell decomposition method, ${ }^{9}$ and so on. In the local navigation, the robot can decide or control its motion and orientation autonomously using equipped sensors such as ultrasonic range finder sensors, sharp infrared range sensors, and vision (camera) sensors, etc. Fuzzy logic, ${ }^{10}$ Neural network, ${ }^{11}$ Neuro-fuzzy, ${ }^{12}$ Genetic algorithm, ${ }^{13}$ Particle swarm optimization algorithm, ${ }^{14}$ Ant colony optimization algorithm, ${ }^{15}$ and Simulated annealing algorithm, ${ }^{16}$ etc. are successfully employed by various researchers to solve the local navigation problem. Rest of the article is organized as follows: Section 2 presents the literature survey of kinematic and dynamic analysis of the wheeled mobile robots. Section 3 discusses the literature review of various soft computing techniques used for mobile robot navigation. Finally, Section 4 describes the summary of this literature survey.

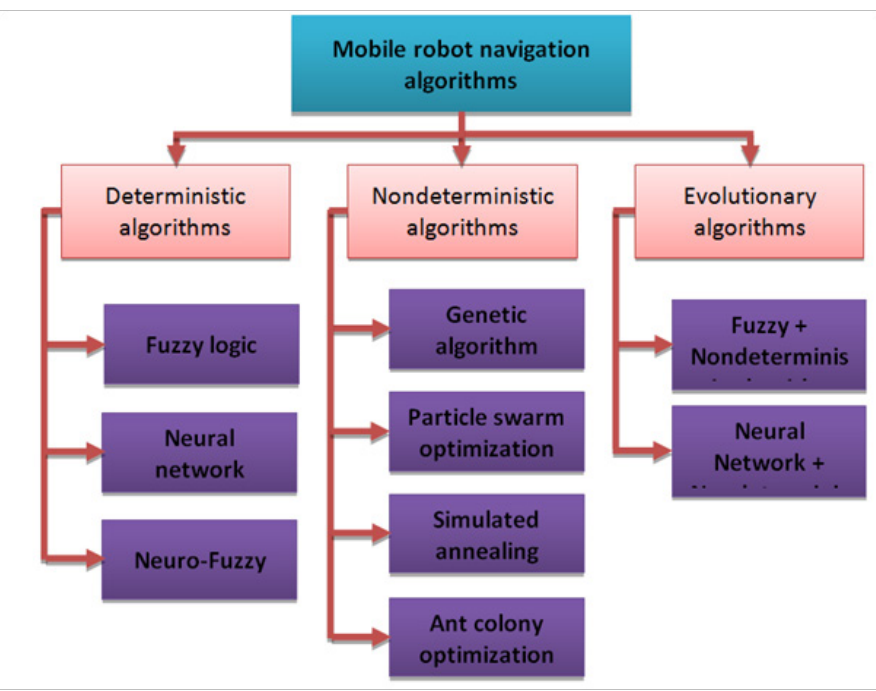

Figure I General classification of the Deterministic algorithm, Nondeterministic (Stochastic) algorithm, and Evolutionary algorithm used for mobile robot navigation.

\section{Study of kinematic and dynamic analysis of the wheeled mobile robot}

The motion control problem of an autonomous wheeled mobile robot has been widely investigated in past decades. In recent years, there has been a growing interest in the design and development of an autonomous wheeled mobile robot using various soft computing techniques. In Hui, ${ }^{17}$ the authors have studied the kinematic and dynamic constraints of a car-like mobile robot and applied it to navigation among moving obstacles in the environments using neurofuzzy approaches. Abadi \& Khooban ${ }^{18}$ have solved the trajectory tracking problem of nonholonomic wheeled mobile robots using Random Inertia Weight Particle Swarm Optimization (RNW-PSO) based optimal Mamdani-type fuzzy controller. The motion problem 
of the wheeled mobile robots on uneven terrain has been addressed in Chakraborty. ${ }^{19}$ Wang \& Yang $^{20}$ have developed the neuro-fuzzy controller for navigation of a nonholonomic differential drive mobile robot. The combination of four sharp infrared sensors is equipped on the robot to read the obstacle distance, and this distance information is fed to the controller to adjust the speed of two separate motors of the robot. Wheeled mobile robots ${ }^{21}$ have been widely used in various industrial applications, transportation, and social sectors, etc. Martinez et al. ${ }^{22}$ have designed the kinematics and dynamics trajectory tracking control of the autonomous unicycle mobile robot using type-2 fuzzy logic and genetic algorithms. An adaptive neural network based motion and orientation control of a nonholonomic wheeled mobile robot has been presented in Al-Arajii. ${ }^{23}$ Liang et al. ${ }^{24}$ have presented the kinematic modelling of the two-wheeled differential drive mobile robot.

\section{Various soft computing techniques used for mobile robot navigation}

In the past few years, many soft computing techniques are proposed by the researchers to solve the robot navigation and obstacle avoidance problem in the various environments. The various soft computing techniques applied for mobile robot navigation in the different static and dynamic environments are summarized below.

\section{Fuzzy logic technique for mobile robot navigation}

The concept of fuzzy logic has been introduced by Zadeh, ${ }^{25}$ which is extensively used in many engineering applications such as mobile robotics, image processing, etc. This method plays a vital role in the field of mobile robots. The fuzzy logic technique has been successfully applied by many researchers to control the position and orientation of mobile robot in the environment. Ren et al. ${ }^{26}$ have designed an intelligent fuzzy logic controller to solve the navigation problem of wheeled mobile robot in an unknown and changing environment. Fuzzy logic systems are inspired by human reasoning, which works based on perception. In Yousfi, ${ }^{27}$ the authors have presented the Gradient method based optimal Takagi-Sugeno fuzzy controller to tune the membership function parameters, and applied it to mobile robot navigation and obstacle avoidance. Qing-yong et al. ${ }^{28}$ have presented the behavior-based fuzzy architecture for mobile robot navigation in unknown environments. They have designed four basic behaviours: goal-seeking behavior, obstacle avoidance behavior, tracking behavior, etc. for mobile robot navigation and tested it in various simulation environments. The eight rule-based fuzzy controllers have been designed by Boubertakh et al. ${ }^{29}$ for obstacle avoidance and goal-seeking behavior of the mobile robot. Muthu et al. ${ }^{30}$ have presented the Atmega microcontroller based fuzzy logic controller for the wheeled mobile robot. The proposed controller train the mobile robot to navigate in an environment without any human intervention. The controller receives inputs (obstacle distance) from the group of sensors to control the right and left motor of the mobile robot.

The sensor-based mobile robot navigation in an indoor environment using a fuzzy logic controller has been discussed ${ }^{31,32} \mathrm{Wu}$ et al.${ }^{33}$ have developed the sensor based mobile robot navigation in the narrow environment using fuzzy controller and genetic algorithm. Where the fuzzy controller provides the initial membership function and the genetic algorithm choose the best membership value to optimize the fuzzy controller for mobile robot navigation. Obstacle avoidance is very important for successful navigation of autonomous mobile robot. Samsudin et al. ${ }^{34}$ have combined the reinforcement learning method and genetic algorithm to optimize the fuzzy controller for improving their performance when the mobile robot moves in an unknown environment. Fuzzy reinforcement learning sensor-based mobile robot navigation has been presented by Beom \& $\mathrm{Cho}^{35}$ for complex environments. Pradhan et al. ${ }^{36}$ have used fuzzy logic controller with different membership functions for the navigation of one thousand robots in an entirely unknown environment. The authors have compared the performance of different membership functions such as triangular, trapezoidal and gaussian for mobile robot navigation and stated that the gaussian membership function is more efficient for navigation. In Liu, ${ }^{37}$ the authors have combined the fuzzy genetic algorithm to solve the path planning and control problem of an autonomous mobile robot (AMR) using ultrasonic range finder sensor information. Farooq et al. ${ }^{38}$ have presented the comparative study between the zero order Takagi-Sugeno and Mamdani-type fuzzy logic models for mobile robot navigation and obstacle avoidance. Both the controllers receive inputs (obstacle distance) from the left and right ultrasonic sensors to control the left and right velocities of the motors of the mobile robot. During comparison study, the authors have found that in terms of smoothness Mamdani-type fuzzy model gives a better result. On the other hand, the Takagi-Sugeno fuzzy model takes less memory space in the real-time microcontroller implementation.

\section{Hybridization of fuzzy and nondeterministic algorithm}

Algabri et al. ${ }^{39}$ have combined the fuzzy logic with other soft computing techniques such as Genetic Algorithm (GA), Neural Networks (NN), and Particle Swarm Optimization (PSO) to optimize the membership function parameters of the fuzzy controller for improving the navigation performance of mobile robot. They have designed two basic fuzzy logic behaviors: Motion to target behavior (MFLC) and obstacle avoidance behavior (AFLC). In Hiu, ${ }^{40}$ the authors have developed genetic-fuzzy and genetic-neural for an adaptive navigation planning of a car-like mobile robot between dynamic obstacles. In this study, the genetic algorithm is employed to adjust the fuzzy membership function and weight of the neural network. Fuzzy PWM (Pulse Width Modulation) controller has been presented in the article ${ }^{41}$ for mobile robot navigation and obstacle avoidance in an unknown environment. Abdessemed et al. ${ }^{42}$ have designed an evolutionary algorithm to optimize the antecedent and consequent parameters of the fuzzy controller, and implemented it for mobile robot path planning. Selekwa et al. ${ }^{43}$ have presented the fuzzy behavior controller for mobile robot navigation in the densely obstacle populated environments. The authors have designed two behavior control actions for navigation, namely obstacle avoidance behavior and the goal-seeking behavior. The obstacle avoidance behavior is done by range finding sensors, which detects the nearest obstacle distance, and the goal-seeking behavior is made by compass measurements, which determines the direction of the goal. Pratihar et al. ${ }^{44}$ have developed a genetic-fuzzy technique based on a combined approach of genetic algorithm and fuzzy logic (GA-FL) to solve the mobile robot motion planning problems in the dynamic environments. Sensor-based wireless fuzzy controller has been designed by Faisal et al. ${ }^{45}$ for mobile robot navigation in the industries among the static and dynamic objects. The two fuzzy controllers: tracking fuzzy logic control (TFLC) and obstacle avoidance fuzzy logic control (OAFLC) are helping the robot to search collision $\square$ free path from the start point to goal point. Babalou \& Seifiour [46] have developed the sensor-based on-line path planning method for the mobile robot in dynamic environments. Li et al. [47] have designed the four types of fuzzy controller: wall-following fuzzy, corner control fuzzy, garageparking fuzzy and parallel-parking fuzzy for the car-like mobile robot 
(CLMR). The developed fuzzy controllers have been implemented real-time using field-programmable gate array (FPGA) chip, and tested it in various experimental scenarios. $\mathrm{Li} \& \mathrm{Chang}^{48}$ have presented a real-time fuzzy target tracking control scheme for autonomous mobile robots using infrared sensors. The behavior-based fuzzy logic controller has been made by Dongshu et al. ${ }^{49}$ to solve the navigation problem of mobile robot in unknown dynamic environment. The different fuzzy rule-based controller has been constructed to deal with different behavior and also helps the robot to get out from the trapped situations. Antonelli et al. ${ }^{50}$ have presented the path-following approach for differential drive mobile robots using the fuzzy logic technique. The designed fuzzy rules are able to emulate the human driving behavior. Ayari et al. ${ }^{51}$ have developed a multi-agent fuzzy logic intelligent control system, which trains the robot to navigate autonomously in dynamic and uncertain environments.

\section{Neural network technique for mobile robot navigation}

The neural network is one of the important technique for the mobile robot navigation. This neural network technique is motivated from the human brain, which is being applied by many researchers in the different fields such as signal and image processing, pattern recognition, mobile robot path planning, and business, etc. Zou et al. ${ }^{52}$ have presented the literature survey of neural networks and its applications in mobile robotics. In Xiao, ${ }^{53}$ the authors have combined the multi-layer feed forward artificial neural network with Q-reinforcement learning method to construct a robust path-planning algorithm for the mobile robot. Rai \& $\mathrm{Rai}^{54}$ have designed the Arduino Uno microcontroller-based DC motor speed control system using the Multilayer neural network controller and Proportional Integral Derivative (PID) controller. Patino \& Carelli ${ }^{55}$ have designed the automatic steering controller for a mobile vehicle using neural network architecture. Yang \& Meng ${ }^{56}$ have applied the biologically inspired neural network to generate a collision-free path in a nonstationary environment. Biologically inspired neural network based wallfollowing mobile robot has been presented by Nichols et al..$^{57}$ Online path planning between unknown obstacles in the environment is an interesting problem in the field of mobile robotics. Motlagh et al. ${ }^{58}$ have presented the target seeking, and obstacle avoidance behaviours using neural networks and reinforcement learning. Mobile robot navigation using hybrid neural network has been addressed by Gavrilov \& Lee. ${ }^{59}$ Singh \& Parhi ${ }^{60}$ have designed multilayer feed forward neural network, which controls the steering angle of the robot autonomously in the static and dynamic environments. The different obstacle distances are the inputs of the four-layered neural network, and the steering angle is the output. Real-time collision-free path planning becomes more difficult when the robot is moving in a dynamic and unstructured environment.

\section{Hybridization of neural network and nondeterministic algorithm}

Rossomando \& Soria ${ }^{61}$ have designed an adaptive neural network PID controller to solve the trajectory tracking control problem of a mobile robot. Al-Jarrah et al. ${ }^{62}$ have described the path planning and coordination of multiple mobile robots using probabilistic neurofuzzy architecture. The authors have applied leader-followers concept to control their position and orientation in the working environment, where the follower robots behave like a leader robot. This proposed probabilistic neuro-fuzzy architecture is the combination of first order Sugeno fuzzy inference model and Adaptive Neuro-Fuzzy Inference System (ANFIS). The fuzzy model has been used to control the linear and angular velocities of the leader robot and the follower robots, and ANFIS is implemented for automatic rule generation from the numerical dataset. In Janglova, ${ }^{63}$ the author has presented a neural network-based technique for intelligent path planning and control of a mobile robot. The two neural network controllers are applied to path planning and control. The first neural network controller helps the robot to search free space in the environment, and the second neural network controller trains the robot for obstacle avoidance. Glasius et al ${ }^{64}$ have used Hopfield neural network for path planning and obstacle avoidance in the complex environment. In $\mathrm{Kin},{ }^{65}$ the authors have proposed type-2 fuzzy neural network (IT2FNN) to solve the obstacle avoidance and position stabilization problems of wheeled mobile robots. IT2FNN consists of three layers: input layer, hidden layers, and output layer. This proposed IT2FNN has four inputs: distance between the robot and goal point, distance between the robot and nearest obstacle, goal angle, and obstacle angle. The outputs of the IT2FNN are linear and angular velocities of the robot. Mahmud et al. ${ }^{66}$ have presented the vision (camera) sensor based Kohonen-type artificial neural network for intelligent navigation of mobile robot. Chohra et al. ${ }^{67}$ have designed intelligent autonomous navigation structure for a vehicle using multi-layered neural networks (NN). Brahmi et al ${ }^{68}$ have solved the path planning and localization problem of mobile robot using recurrent neural network (RNN). This RNN allows the robot to navigate autonomously in the unknown environments. In Yang, ${ }^{69}$ the authors have controlled the torque dynamic of nonholonomic mobile robot using neural network architecture.

\section{Neuro-fuzzy technique for mobile robot navigation}

Zhu \& Yang ${ }^{12}$ have presented a neuro-fuzzy sensor based reactive navigation of mobile robots in unknown environments. Fortyeight Fuzzy rules and two behaviours, target seeking, and obstacle avoidance are designed using this model. A neural network based learning techniques is developed to tune the parameters of membership functions, which reduces the navigation path length from a start position to the end position in an environment. Al Mutib \& Mattar ${ }^{70}$ have proposed the sensor-based navigation of mobile robot using neuro-fuzzy architecture. The authors have used eight ultrasonic range finder sensors for surrounding obstacle detection as the input of the neuro-fuzzy controller for selecting the correct left and the right wheel speeds for a mobile robot. Godjevac \& Steele ${ }^{71}$ have integrated the Takagi-Sugeno type fuzzy controller and Radial basis function neural network (RBFNN) to solve the mobile robot path planning. Where, the fuzzy logic is used to handle the uncertainty of the environment, and the neural network is used to tune the parameters of membership functions. In $\mathrm{Li}^{72}$ the authors have constructed behaviour-based neuro-fuzzy control architecture for a mobile robot navigation in an unstructured environment. The neural network is used to train the robot to reach the goal, and fuzzy architecture is integrated with it to control the velocities of the robot.

Joshi \& Zaveri $^{73}$ have developed a neuro-fuzzy system for reactive navigation and control of a mobile robot in the environment with the presence of static and dynamic obstacles. Marichal et al. ${ }^{74}$ have designed a neuro-fuzzy sensor-actuator control technique to steer the mobile robot in unknown environments. RAM based neuro-fuzzy approach for mobile robot navigation has been presented by Zhang et al. $^{75}$ They have used the fuzzy rule-based controller to interpret sensory information, and neural network controls the heading angle of the robot during navigation. Baturone et al. ${ }^{76}$ have designed a low-cost embedded neuro-fuzzy controller for navigation of car-like mobile robot between the obstacles. Ma et al. ${ }^{77}$ have used mixed soft computing techniques like fuzzy inference system and neural network to improve the learning and decision-making speed of a robot in 
unknown environments. Imen et al. ${ }^{78}$ have applied the Adaptive NeuroFuzzy Inference System (ANFIS) technique to solve the path tracking problem of the nonholonomic wheeled mobile robots. They have used gradient descent learning algorithm to adjust the membership function parameters of the ANFIS. In Ganapathy, ${ }^{79}$ the authors have designed the two controllers: a Fuzzy Logic (FL) controller for obstacle avoidance and Artificial Neural Network (ANN) for wall-following of the mobile robot. Both the controllers receive inputs from the different sensors to avoid the obstacles when the robot moves towards the desired goal. Zhao \& Wang ${ }^{80}$ have incorporated sonar sensors with the neural network to solve the navigation problem of the autonomous mobile robot.

Kumar \& Dhama et al. ${ }^{81}$ have integrated the neural network and fuzzy logic to control the motion and orientation of the mobile robot in the crowded unknown environment. In their work, the authors have used fuzzy rule-based and neural network for goal reaching and actuator control, respectively. Song et al.$^{82}$ have designed a heuristic fuzzy-neuro network to create a mapping between the ultrasonic sensor data and velocity command of the robot. They have used sixteen rules to control the direction of the mobile robot. In Lee, ${ }^{83}$ the authors have developed a Takagi-Sugeno type recurrent neuro fuzzy system and hybrid algorithm (genetic algorithm with particle swarm optimization) to improve the path tracking stability of the mobile robots. The neuro-fuzzy systems have been classified into two categories: ${ }^{84}$ adaptive neuro-fuzzy systems (ANFIS) and hybrid neuro-fuzzy systems. Deshpande \& Bhosale ${ }^{84}$ have discussed the navigation of a nonholonomic wheeled mobile robot using ANFIS controller. Rusu \& Petriu et al ${ }^{85}$ have presented a sensor-based neurofuzzy controller for mobile robot navigation in indoor environments. They have used infrared and contact sensors for target seeking and obstacle avoidance behavior. Pothal \& Parhi ${ }^{86}$ have proposed a sensor based adaptive neuro-fuzzy inference controller for navigation of single and multiple mobile robots in the highly cluttered environment. The authors have designed control architecture, which is able to avoid obstacle autonomously in various situations and reach the target efficiently. Neural network integrated fuzzy controller has been designed by $\mathrm{Ng} \&$ Trivedi $^{87}$ for mobile robot navigation and wallfollowing control. In their work, the authors have used only five rules to control the steering angle, heading direction, and speed of the robot during wall-following. Demirli \& $\mathrm{Khoshnejad}^{88}$ have developed sensor-based neuro-fuzzy controller for autonomous parallel parking of a car-like mobile robot (CLMR). The proposed model received data from the sonar sensors to control the turning angle of CLMR. Al-Mayyahi et al. ${ }^{89}$ have applied ANFIS technique for autonomous ground vehicle (AGV) navigation. In this work, they have designed four ANFIS controllers to control the left and right angular velocities, and angle between the robot and target (heading angle). In Pradhan, ${ }^{90}$ the authors have designed a navigational approach for multiple mobile robots using a neuro-fuzzy controller. The proposed controller receives input (obstacle distance) from the array of sensors to actuate the left and right wheel velocities of the mobile robots. Algabri et al. ${ }^{91}$ have applied ANFIS controller for mobile robot navigation and obstacle avoidance in an unknown environment. The authors have presented many simulation tests using Khepera Simulator (KiKs).

\section{Genetic algorithm for mobile robot navigation}

Ghorbani et al. ${ }^{13}$ have solved the global path planning problem of a mobile robot in the complex environment using genetic algorithm approach. Elshamli et al. ${ }^{92}$ have presented a genetic algorithm technique for solving the path planning problem of a mobile robot in static and dynamic environments. Mohanta et al. ${ }^{93}$ have designed Petri-GA technique to optimize the navigation path length of multiple mobile robots in the cluttered environment. Kubota et al. ${ }^{94}$ have used the fuzzy controller to guide the mobile robot in a static and dynamic environment, and the conventional genetic algorithms (GAs) are integrated with it, to optimize the navigation path length. Tuncer \& Yildirim ${ }^{95}$ have proposed a new mutation operator for a genetic algorithm $(\mathrm{GA})$ and applied it for mobile robot navigation in the dynamic environments. Moreover, the authors have tested their developed method in various simulation environments and compared it with traditional GA techniques and stated that their developed mutation operator based GA performs better over traditional GA. In Ming ${ }^{96}$ the authors have designed a genetic algorithm to choose the best membership parameters from the fuzzy inference system and implemented it to control the steering angle of a mobile robot in the partially unknown environment. Hu et al. ${ }^{97}$ have designed the knowledge-based genetic algorithm for mobile robot navigation between U-shaped obstacle and maze environment.

Liu et al. ${ }^{98}$ have presented the optimal path planning technique for a mobile robot using fuzzy logic and genetic algorithm. The fuzzy controllers are applied to modify the moving direction of the mobile robot according to the obstacle distance received from the sensors, and genetic algorithm is used to adjust and tune membership function and rules. Improved genetic algorithm based mobile robot navigation has been proposed by $\mathrm{Li}$ et al. ${ }^{99}$ The authors have done many simulation tests in the both static and dynamic environments to show the effectiveness of the proposed algorithm. Qu et al. ${ }^{100}$ have developed the improved genetic algorithm instead of a conventional genetic algorithm for global path planning of the multiple mobile robots. The advantages of the improved genetic algorithm are capable of guiding the mobile robots efficiently from the starting node to end node without any collision in the environment. In Algabri, ${ }^{101}$ the authors have implemented Genetic-Fuzzy Controller (GA-FLC) to optimize and tune the Gaussian membership function parameters for mobile robot motion control. Castillo et al. ${ }^{102}$ have designed Multiple Objective Genetic Algorithm (MOGA) for navigation path optimization of the mobile robot. Arora et al. ${ }^{103}$ have presented the single fitness based genetic algorithm for solving the navigation problem in the dynamic environments. They have designed a fitness function based on the Euclidean distance formula between the robot and obstacle.

\section{Simulated annealing algorithm for mobile robot navigation}

The concept of simulated annealing algorithm has come from statistical mechanics. ${ }^{104}$ The simulated annealing is an iterative search algorithm inspired by the annealing of metals. ${ }^{105}$ Miao \& Tian ${ }^{16}$ have applied the heuristic method based simulated annealing algorithm for robot path planning in the dynamic environments. The authors have compared this proposed algorithm to the Dijkstra algorithm and stated that the proposed algorithm consumes less processing time to get a solution compared to Dijkstra algorithm. Sensor-based autonomous navigation of a mobile robot in the dynamic environment has been presented by Chang \& Song. ${ }^{106}$ Martinez-Alfaro et al. ${ }^{107}$ have developed the simulated annealing and fuzzy logic for designing an automatic path planning technique for mobile robot. The simulated annealing algorithm is used to search a collision-free optimal trajectory between the fixed polygonal obstacles, and forty-nine fuzzy rules are applied to adjust the velocity of the robot during navigation. Zhu et al. ${ }^{108}$ have presented the global path planning 
method for a mobile robot using Artificial Potential Field (APF) method and Simulated Annealing Algorithm (SAA). In Precup, ${ }^{109}$ the authors have used SAA with fuzzy logic to adjust and optimize the antecedent and the consequent parameters of the fuzzy membership function and applied it to solve the optimization problem of the servo systems. Janabi-Sharifi \& Vinke ${ }^{110}$ have addressed the local and global navigation problems in the real environment using Artificial Potential Field method and Simulated Annealing Algorithm. Tavares et al. ${ }^{111}$ have discussed the off-line path planning problem of a mobile robot using SAA. They have designed some adaptive tuning parameters to change the behavior of that algorithm. Due to the slow convergence rate of the conventional simulated annealing algorithm, the Liang \& $\mathrm{Xu}^{112}$ have presented a modified simulated annealing algorithm, and applied it to mobile robot global path planning.

Nakamura \& Kehtarnavaz ${ }^{113}$ have designed an optimal fuzzy logic controller for autonomous mobile robot navigation and hurdle avoidance using a genetic algorithm and SAA combinatorial optimization techniques. Hussein et al. ${ }^{114}$ have designed three metaheuristic optimization algorithms: Tabu Search, Simulated Annealing and Genetic Algorithm; and implemented these algorithms to improve the navigation performance of mobile robot from the start point to goal point in an environment. Miao \& Tian ${ }^{115}$ have presented a simulated annealing algorithm based intelligent navigational controller, which helps the robot to search an optimal or near-optimal path in the static and dynamic environments. Zhang et al. ${ }^{116}$ have combined the simulated annealing algorithm and Ant Colony Optimization (ACO) algorithm to increase the navigation speed of the mobile robot. In Gao, ${ }^{117}$ the authors have improved the convergence speed of the simulated annealing algorithm using the artificial neural network and applied it to mobile robot path planning. Synodinos \& Aspragathos ${ }^{118}$ have integrated simulated annealing algorithm and artificial potential field method to rescue the robot from undesired local minima problem during navigation. Zhao \& $\mathrm{Zu}^{119}$ have developed a Modified Particle Swarm Optimization (MPSO) technique for mobile robot navigation in the dynamic environment.

\section{Particle Swarm Optimization Algorithm for Mobile Robot Navigation}

Particle swarm optimization (PSO) is a population-based stochastic algorithm, which is inspired by the social behavior of bird flocks. PSO algorithm is used to find an optimal or near optimal solution of the problem using fitness function $\mathrm{f}(\mathrm{x})=\mathrm{f}\left(\mathrm{x}_{1}, \mathrm{x}_{2}, \mathrm{x}_{3}, \ldots . . \mathrm{x}_{\mathrm{n}}\right)$, where $x_{i}$ is a population of the particles. Ahmadzadeh \& Ghanavati ${ }^{14}$ have presented the PSO algorithm based navigation method for multiple mobile robots. The robots move according to the global best (g-best) position of a particle in every iteration. To prepare an optimal intelligent controller for an autonomous wheeled mobile robot, the Castillo et al. ${ }^{120}$ have designed the hybridization of an Ant Colony Optimization (ACO) algorithm and the Particle Swarm Optimization (PSO) algorithm to optimize the membership function of a fuzzy controller. Zhang et al. ${ }^{121}$ have proposed the Multi-Objective Particle Swarm Optimization Algorithm (MOPSO) to search a collision-free optimal path in the uncertain dynamic environment. Zhang \& $\mathrm{Li}^{122}$ have presented a new objective function for mobile robot navigation using PSO. This objective function works based on the position of the obstacles and target in the environment. PSO algorithm has been successfully applied by Raja \& Pugazhenthi ${ }^{123}$ to optimize the travel time of the mobile robot in the dynamic environments. This algorithm searches the feasible path in the environment by randomly in every iteration. Masehian \& Sedighizadeh ${ }^{124}$ have solved the motion planning problem of the mobile robot by using multi-objective PSO.

PSO-based optimal fuzzy controller has been designed by Wong et al. ${ }^{125}$ to determine the velocities of the left-wheeled motor and rightwheeled motor of the differential drive mobile robot. Specialized particle swarm optimization algorithm has been presented by Li et al. ${ }^{126}$ for global optimum path planning of mobile robots. The authors have conducted many simulation tests in the simple and complicated environment to show the effectiveness of the proposed algorithm. Huang ${ }^{127}$ has designed the Parallel Met heuristic Particle Swarm Optimization (PPSO) algorithm to solve the global path planning problem of an autonomous mobile robot. The author has implemented this PPSO algorithm in real-time using the field-programmable gate array (FPGA) chip. Chung et al. ${ }^{128}$ have developed PSO and fuzzy based combinatorial algorithm to design intelligent navigation architecture for a mobile robot. They have used PSO algorithm to escape the robot from the dead-end condition, and the fuzzy algorithm is used to control the turn angle of a wheeled mobile robot during navigation and obstacle avoidance. Shiltagh \& Jala ${ }^{129}$ have investigated the application of Modified Particle Swarm Optimization (MPSO) in the field of mobile robotics to determine a shortest feasible path from the beginning to end in an environment between obstacles. The developed modified PSO increases the convergence rate of the algorithms. Chatterjee \& Matsuno ${ }^{130}$ have solved the Simultaneous Localization and Mapping (SLAM) problem of mobile robots or vehicle using modified PSO and fuzzy evolutionary algorithm. Juang $\&$ Chang ${ }^{131}$ have presented an evolutionary-group-based particleswarm-optimization (EGPSO) for automatic learning of fuzzy system for mobile robot navigation or wall-following control in unknown environments. In $\mathrm{Lu},{ }^{132}$ the authors have converted the robot path planning problem to the minimization problem and designed a fitness function based on the positions of the target and obstacles in the environment. Allawi \& Abdalla ${ }^{133}$ have proposed the sensor based PSO-fuzzy type-2 model for the navigation of multiple mobile robots. They have used PSO algorithm to determine the optimal input/ output membership function parameters and rules for the fuzzy type- 2 controller.

\section{Ant colony optimization algorithm and other nondeterministic algorithms for mobile robot navigation}

The Ant Colony Optimization (ACO) algorithm is used by many authors for mobile robot navigation and obstacle avoidance in the different environments. ACO is a probabilistic algorithm proposed by Dorigo et al. ${ }^{134}$ in 1999, which is originated from bionics. Guan-Zheng et al. ${ }^{135}$ have presented the modern global path planning method for a mobile robot by applying Ant Colony System (ACS) algorithm and the Dijkstra algorithm. Purian \& Sadeghian ${ }^{136}$ have explored the optimal path for a mobile robot in an unknown dynamic environment using Ant Colony Optimization (ACO) algorithm and fuzzy controller. This ACO algorithm searches the optimal value from the fuzzy rule table and minimizes the distance between the start points to goal point of the mobile robot with obstacle avoidance competence. Bi et al. ${ }^{137}$ have designed an Ant Colony System (ACS) to improve the path searching speed of the mobile robot in the dynamic environment. Dong et al. ${ }^{138}$ have presented an improved ACO algorithm for obstacle avoidance of mobile robot in the grid environment. In Ganapathy, ${ }^{139}$ the authors have described various behaviours such as goal-seeking, wall-following obstacle avoidance for mobile robot navigation using improved ACO algorithm. Fan et al. ${ }^{140}$ have applied an intensified ant colony optimization (ACO) algorithm to search an optimal path for 
mobile robot between irregular obstacles in an environment. Sariff $\&$ Buniyamin ${ }^{141}$ have compared the performances of GA and ACO algorithm for robot path planning in the global static environment and stated that the ACO algorithm takes less time to search an optimal path in the environment compared to GA. Hsu et al. ${ }^{142}$ have proposed an improved ant colony system algorithm by including a new pheromone updating parameter for path planning of mobile robots. Ganganath et al. ${ }^{143}$ have designed an off-line path planner for nonholonomic mobile robots using an ACO algorithm. Juang \& $\mathrm{Hsu}^{144}$ have designed the reinforcement ant optimized fuzzy controller (RAOFC) and applied it for wheeled mobile robot wall-following control under reinforcement learning environments. The inputs of the proposed controller are range-finding sonar sensors, and the output is a robot steering angle. The antecedent and consequent parts of the fuzzy controller have aligned by the fuzzy type- 2 clustering and ACO respectively.

Hsu \& Juang ${ }^{145}$ have designed the wall-following mobile robot using a type-2 fuzzy controller (IT2FC) and integrated it with an ACO algorithm to improve the performance of the controller. The steering angle and moving speed of the wall-following mobile robot has been controlled by two type- 2 fuzzy controllers. In Juang, ${ }^{146}$ the authors have presented the navigation method of the two robots (a leader robot and a follower robot) using fuzzy controllers (FC). They have applied continuous ant colony optimization and particle swarm optimization (AF-CACPSO) to the control the mobile robots to perform obstacle boundary following behavior. Hsu \& Juang ${ }^{147}$ have adopted the multi-objective ACO for optimized the rule parameters of the fuzzy controller (FC) for wall-following mobile robot. Chen et al. ${ }^{148}$ have designed a scent pervasion (pheromone) principle of ant (ACO) based robotic path planning in a map environment. Hossain \& Ferdousand ${ }^{149}$ have applied Bacterial Foraging Optimization (BFO) method for mobile robot navigation to find out shortest possible path within the minimum time from the start position to the goal position between moving obstacles. Liang et al. ${ }^{150}$ have developed a bacterial foraging algorithm for making a bio-inspired path planning strategy for a mobile robot. In the proposed model, the behavior of bacteria is applied to search an optimal collision-free path between the start nodes to the target node in an environment with obstacles. Brand \& $\mathrm{Yu}^{151}$ have applied the Firefly Algorithm (Glow-worm swarm optimization) to find a collision free shortest path in the two-dimensional static and dynamic environment for a mobile robot. They have compared this proposed algorithm to ACO algorithm and stated that the proposed algorithm provides better results (in terms of path length and computational cost) compared to ACO algorithm. Mohajer et al. ${ }^{152}$ have presented a new Random Particle Optimization Algorithm (RPOA), which is inspired by the bacterial foraging technique, and used for local path planning for mobile robots in the dynamic and unknown environments. The proposed algorithm randomly searches the feasible path in the environment and avoids the moving obstacles by using the sensors. In Luo, ${ }^{153}$ the authors have presented a review paper of multi-sensor fusion and integration and its application in the field of Mechatronic.

\section{Summary}

This article provides a literature survey of various techniques employed for mobile robot navigation. After summarizing the above literature review, the major conclusions are listed below:

a. The various soft computing techniques e.g. Deterministic, Nondeterministic, and Evolutionary algorithms, etc. have been applied by the researchers for mobile robot navigation and obstacle avoidance in the different environments. b. According to literature survey, most of the researchers have used these soft computing techniques for mobile robot navigation and obstacle avoidance in only static environments. However, few researchers have considered dynamic environments for mobile robot navigation.

c. From the literature survey, it is observed that many researchers have demonstrated only computer simulation results without implementations of physical robot.

d. Nature-inspired algorithm based mobile robot navigation and obstacle avoidance is an important topic for the research. The hybridization of Deterministic and Nondeterministic algorithms is also a better choice for the research.

\section{Acknowledgments}

None.

\section{Conflict of interest}

Author declares that there is none of the conflicts.

\section{References}

1. Ni J, Wu L, Fan X, Yang SX. Bioinspired Intelligent Algorithm and Its Applications for Mobile Robot Control: A Survey. Comput Intell Neurosci. 2016. p. 1-15.

2. Takahashi O, Schilling RJ. Motion Planning in a Plane Using Generalized Voronoi Diagrams. IEEE Robotics and Automation. 1989;5(2):143-150.

3. Bhattacharya P, Gavrilova ML. Roadmap-Based Path Planning-Using the Voronoi Diagram for a Clearance-Based Shortest Path. IEEE Robotics and Automation. 2008;15(2):58-66.

4. Gomez EJ, Martinez Santa F, Martinez Sarmiento FHA. Comparative Study of Geometric Path Planning Methods for a Mobile Robot: Potential Field and Voronoi Diagrams. In: IEEE International Congress of Engineering Mechatronic and Automation (CIIMA), Colombia. 2013. p. $1-6$.

5. Abiyev R, Ibrahim D, Erin B. Navigation of Mobile Robots in the Presence of Obstacles. Advances in Engineering Software. 2010;41(10):11791186 .

6. Soltani AR, Tawfik H, Goulermas JY, et al. Path Planning in Construction Sites: Performance Evaluation of the Dijkstra A and GA Search Algorithms. ELSEVIER Advanced Engineering Informatics. 2002;16(4):291-303.

7. Masehian E, Amin-Naseri MR. A Voronoi Diagram-Visibility GraphPotential Field Compound Algorithm for Robot Path Planning. Journal of Robotic System. 2004;21(6):275-300.

8. Weigl M, Siemiaatkkowska B, Sikorski KA, et al. Grid-Based Mapping for Autonomous Mobile Robot. ELSEVIER Robotics and Autonomous Systems. 1993;11(1):13-21.

9. Park KH, Kim YJ, Kim JH. Modular Q-Learning based Multi-Agent Cooperation for Robot Soccer. ELSEVIER Robotics and Autonomous Systems. 2001;35(2):109-122.

10. Montaner MB, Ramirez-Serrano A. Fuzzy Knowledge-Based Controller Design for Autonomous Robot Navigation. ELSEVIER Expert Systems with Applications. 1998;14(1):179-186

11. Engedy I, Horvath G. Artificial Neural Network based Local Motion Planning of a Wheeled Mobile Robot. IEEE International Symposium on Computational Intelligence and Informatics (CINTI). 2010. p. 213-218.

12. Zhu A, Yang SX. Neurofuzzy-Based Approach to Mobile Robot Navigation in Unknown Environments. IEEE Transactions on Systems. 2007;37(4):610-621. 
13. Ghorbani A, Shiry S, Nodehi A.Using Genetic Algorithm for a Mobile Robot Path Planning. IEEE International Conference on Future Computer and Communication. 2009. p. 164-166.

14. Ahmadzadeh S, Ghanavati M. Navigation of Mobile Robot Using the PSO Particle Swarm Optimization. Journal of Academic and Applied Studies (JAAS). 2012;2(1):32-38.

15. Garcia MP, Montiel O, Castillo O, et al. Path Planning for Autonomous Mobile Robot Navigation with Ant Colony Optimization and Fuzzy Cost Function Evaluation. Applied Soft Computing. 2009;9(3):1102-1110.

16. Miao H, Tian YC. Dynamic Robot Path Planning using an Enhanced Simulated Annealing Approach. ELSEVIER Applied Mathematics and Computation. 2013;222:420-437.

17. Hui NB, Mahendar V, Pratihar DK. Time-Optimal, and Collision-Free Navigation of a Car-Like Mobile Robot using Neuro-Fuzzy Approaches. ELSEVIER Fuzzy Sets and Systems. 2006;157(16):2171-2204.

18. Abadi DNM and Khooban MH. Design of Optimal Mamdani-Type Fuzzy Controller for Nonholonomic Wheeled Mobile Robots. Journal of King Saud University-Engineering Sciences. 2015;27(1):92-100.

19. Chakraborty N, Ghosal A. Kinematics of Wheeled Mobile Robots on Uneven Terrain. ELSEVIER Mechanism and Machine Theory. 2004;39(12):1273-1287.

20. Wang X, Yang SXA. Neuro-Fuzzy Approach to Obstacle Avoidance of a Nonholonomic Mobile Robot. IEEE/ASME International Conference on Advanced Intelligent Mechatronic. 2003. p. 29-34.

21. Das T, Kar IN, Chaudhury S. Simple Neuron-Based Adaptive Controller for a Nonholonomic Mobile Robot Including Actuator Dynamics. Neurocomputing. 2006;69(16):2140-2151.

22. Martinez R, Castillo O, Aguilar. LT Optimization of Interval Type-2 Fuzzy Logic Controllers for a Perturbed Autonomous Wheeled Mobile Robot using Genetic Algorithms. ELSEVIER Information Sciences. 2009;179(13):2158-2174.

23. Al-Araji AS, Abbod MF, Al-Raweshidy HS. Applying Posture Identifier in Designing an Adaptive Nonlinear Predictive Controller for Nonholonomic Mobile Robot. Neurocomputing. 2013;99:543-554.

24. Liang Y, Xu L, Wei R, et al. Adaptive Fuzzy Control for Trajectory Tracking of Mobile Robot. IEEE/RSJ International Conference on Intelligent Robots and Systems (IROS). 2010. p. 4755-4760.

25. Zadeh LA. The Concept of a Linguistic Variable and its Application to Approximate Reasoning-I. ELSEVIER Information Science. 1975;8(3):199-249.

26. Ren L, Wang W, Du Z. A New Fuzzy Intelligent Obstacle Avoidance Control Strategy for Wheeled Mobile Robot. IEEE International Conference on Mechatronics and Automation (ICMA). 2012. p. 17321737.

27. Yousfi N, Rekik C, Jallouli M, et al. Optimized Fuzzy Controller for Mobile Robot Navigation in a Cluttered Environment. IEEE 7th International Multi-Conference on Systems, Signals and Devices. 2010. p. $1-7$.

28. Qing-yong B, Shun-ming L, Wei-yan S, et al. A Fuzzy Behavior-Based Architecture for Mobile Robot Navigation in Unknown Environments. IEEE International Conference on Artificial Intelligence and Computational Intelligence. 2009. p. 257-261.

29. Boubertakh H, Tadjine M, Glorennec P, et al. A Simple Goal Seeking Navigation Method for a Mobile Robot using Human Sense, Fuzzy Logic and Reinforcement Learning. Journal of Automatic Control. 2008;18(1):23-27.

30. Muthu T, Thierry Gloude R, Swaminathan S, et al. Fuzzy Logic Controller for Autonomous Navigation. IEEE International Conference on Communications and Signal Processing (ICCSP). 2012. p. 81-92.
31. El-Teleity SAL, Nossair ZB, et al. Fuzzy Logic Control of an Autonomous Mobile Robot. IEEE 16th International Conference on Methods and Models in Automation and Robotics (MMAR). 2011. p. 188-193.

32. Raguraman SM, Tamilselvi D, Shivakumar N. Mobile Robot Navigation Using Fuzzy logic Controller. IEEE 16th International Conference on Control, Automation, Communication and Energy Conservation. 2009. p. $1-5$.

33. Wu S, Li Q, Zhu E, et al. Fuzzy Controller of Pipeline Robot Navigation Optimized by Genetic Algorithm. IEEE International Conference on Control and Decision Conference (CCDC). 2008. p. 904-908.

34. Samsudin K, Ahmad FA, Mashohor S. A Highly Interpretable Fuzzy Rule Base using Ordinal Structure for Obstacle Avoidance of Mobile Robot. Applied Soft Computing. 2011;11(2):1631-1637.

35. Beom HR, Cho KS. A Sensor-Based Navigation for a Mobile Robot using Fuzzy Logic and Reinforcement Learning. IEEE Transactions on Systems, Man, and Cybernetics. 1995;25(3):464-477.

36. Pradhan SK, Parhi DR, Panda AK. Fuzzy Logic Techniques for Navigation of Several Mobile Robots. Applied Soft Computing. 2009;9(1):290-304.

37. Liu Q, Lu YG, Xie CX. Optimal Genetic Fuzzy Obstacle Avoidance Controller of Autonomous Mobile Robot Based on Ultrasonic Sensors. IEEE International Conference on Robotics and Biomimetics. 2006. p. $125-129$.

38. Farooq U, Hasan KM, Abbas G, et al. Comparative Analysis of Zero Order Sugeno and Mamdani Fuzzy Logic Controllers for Obstacle Avoidance Behavior in Mobile Robot Navigation. IEEE International Workshop on Current Trends in Information Technology (CTIT). 2011. p. 113-119.

39. Algabri M, Mathkour H, Ramdane H, et al. Comparative Study of Soft Computing Techniques for Mobile Robot Navigation in an Unknown Environment. Computers in Human Behavior. 2015;50:42-56.

40. Hui NB, Pratihar DK. A Comparative Study on Some Navigation Schemes of a Real Robot Tackling Moving Obstacles. Robotics and Computer-Integrated Manufacturing. 2009;25(4):810-828.

41. Rusu CG, Birou IT, Szoke E. Fuzzy Based Obstacle Avoidance System for Autonomous Mobile Robot. IEEE International Conference on Automation Quality and Testing Robotics (AQTR). 2010. p. 1-6.

42. Abdessemed F, Benmahammed K, Monacelli E. A Fuzzy-Based Reactive Controller for a Non-Holonomic Mobile Robot. ELSEVIER Robotics and Autonomous Systems. 2004;47(1):31-46.

43. Selekwa MF, Dunlap DD, Shi D, et al. Robot Navigation in Very Cluttered Environments by Preference-Based Fuzzy Behaviors. Robotics and Autonomous Systems. 2008;56(3):231-246.

44. Pratihar DK, Deb K, Ghosh A. A Genetic-Fuzzy Approach for Mobile Robot Navigation among Moving Obstacles. International Journal of Approximate Reasoning. 1999;20(2):145-172.

45. Faisal M, Hedjar R, Al Sulaiman M, et al. Fuzzy Logic Navigation and Obstacle Avoidance by a Mobile Robot in an Unknown Dynamic Environment. INTECH International Journal of Advanced Robotic Systems. 2013;10(1):1-7.

46. Babalou A, Seifiour N. Application of Fuzzy Decision Making in Mobile Robot Navigation in Dynamic Environments. IEEE International Conference on Fuzzy Systems. 2009. p. 877-881.

47. Li THS, Chang SJ, Chen YX. Implementation of Human-Like Driving Skills by Autonomous Fuzzy Behavior Control on an FPGA-Based Car-Like Mobile Robot. IEEE Transactions on Industrial Electronics. 2003;50(5):867-880.

48. Li THS, Chang SJ, Tong W. Fuzzy Target Tracking Control of Autonomous Mobile Robots by Using Infrared Sensors. IEEE Transactions on Fuzzy Systems. 2004;12(4):491-501. 
49. Dongshu W, Yusheng Z, Wenjie S. Behavior-Based Hierarchical Fuzzy Control for Mobile Robot Navigation in Dynamic Environment. IEEE International Conference on Control and Decision Conference (CCDC). 2011. p. 2419-2424.

50. Antonelli G, Chiaverini S, Fusco G. A Fuzzy-Logic-Based Approach for Mobile Robot Path Tracking. IEEE Transactions on Fuzzy Systems. 2007;15(2):211-221.

51. Ayari E, Hadouaj S, Ghedira K. A Fuzzy Logic Method for Autonomous Robot Navigation in Dynamic and Uncertain Environment Composed with Complex Traps. IEEE 5th International Multi-conference on Computing in the Global Information Technology. 2010. p. 18-23.

52. Zou AM, Hou ZG, Fu SY. Neural Networks for Mobile Robot Navigation: a Survey. Advances in Neural Networks. 2006. p. 1218-1226.

53. Xiao H, Liao L, Zhou F. Mobile Robot Path Planning Based on Q-ANN. IEEE International Conference on Automation and Logistics. 2007. p. 2650-2654.

54. Rai N, Rai B. Neural Network based Closed loop Speed Control of DC Motor using Arduino Uno. International Journal of Engineering Trends and Technology. 2013;4(2):137-140.

55. Patino HD, Carelli R. Neural Network-Based Optimal Control for Autonomous Mobile Vehicle Navigation. IEEE International Symposium on Intelligent Control. 2004. p. 391-396.

56. Yang SX, Meng M. Neural Network Approaches to Dynamic CollisionFree Trajectory Generation. IEEE Transactions on Systems, Man, and Cybernetics Applications and Reviews. 2001;31(3):302-318.

57. Nichols E, McDaid LJ, Siddique N. Biologically Inspired SNN for Robot Control. IEEE Trans Cybern. 2013;15(2):115-128.

58. Motlagh O, Nakhaeinia D, Tang SH, et al. Automatic Navigation of Mobile Robots in Unknown Environments. Neural Computing and Applications. 2014;24(7):1569-1581.

59. Gavrilov AV, Lee S. An Architecture of Hybrid Neural Network Based Navigation System for Mobile Robot. IEEE Seventh International Conference on Intelligent Systems Design and Applications. 2007. p. 587-590.

60. Singh MK, Parhi DR. Path Optimisation of a Mobile Robot Using an Artificial Neural Network Controller. International Journal of Systems Science. 2011;42(1):107-120.

61. Rossomando FG, Soria CM . Design and Implementation of Adaptive Neural PID for Non Linear Dynamics in Mobile Robots. IEEE Latin America Transactions. 2015;13(4):913-918.

62. Al-Jarrah R, Shahzad A, Roth H. Path Planning and Motion Coordination for Multi-Robots System Using Probabilistic Neuro-Fuzzy. ELSEVIER IFAC-Papers Online. 2015;48(10):46-51

63. Janglova D (2004) Neural Networks in Mobile Robot Motion. International Journal of Advanced Robotic Systems 1(1): 15-22.

64. Glasius R, Komoda A, Gielen SC. Neural Network Dynamics for Path Planning and Obstacle Avoidance. Neural Networks. 1995;8(1):125-133.

65. Kim CJ, Chwa D. Obstacle Avoidance Method for Wheeled Mobile Robots Using Interval Type-2 Fuzzy Neural Network. IEEE Transactions on Fuzzy Systems. 2015;23(3):677-687.

66. Mahmud F, Arafat A, Zuhori ST. Intelligent Autonomous Vehicle Navigated by Using Artificial Neural Network. IEEE International Conference on Electrical and Computer Engineering. 2012. p. 105-108.

67. Chohra A, Farah A, Benmehrez C. Neural Navigation Approach for Intelligent Autonomous Vehicles (IAV) in Partially Structured Environments. Applied Intelligence. 1998;8(3):219-233.

68. Brahmi H, Ammar B, Alimi AM. Intelligent Path Planning Algorithm For Autonomous Robot Based on Recurrent Neural Networks. IEEE
International Conference on Advanced Logistics and Transport (ICALT). 2013. p. 199-204.

69. Yang SX, Hu T, Yuan X, et al. Neural Network based Torque Controller for Collision-Free Navigation of Mobile Robots. IEEE International Conference on Robotics and Automation (ICRA). 2003. p. 13-18.

70. Al Mutib K, Mattar E. Neuro-fuzzy Controlled Autonomous Mobile Robotics System. IEEE 13th International Conference on Modelling and Simulation. 2011. p. 1-7.

71. Godjevac J, Steele N. Neuro-Fuzzy Control of a Mobile Robot ELSEVIER Neurocomputing. 1999;28(1):127-143.

72. Li W, Ma C, Wahl FM. A Neuro-Fuzzy System Architecture for Behavior-Based Control of a Mobile Robot in Unknown Environments. Fuzzy Sets and Systems. 1997;87(2):133-140.

73. Joshi MM, Zaveri M. Neuro-Fuzzy Based Autonomous Mobile Robot Navigation System. IEEE 11th International Conference Control, Automation, Robotics and Vision. 2010. p. 384-389.

74. Marichal GN, Acosta L, Moreno L, et al. Obstacle Avoidance for a Mobile Robot: A Neuro-Fuzzy Approach. ELSEVIER Fuzzy Sets and Systems. 2001;124(2):171-179.

75. Zhang N, Beetner D, Wunsch DC, et al. An Embedded Real-Time NeuroFuzzy Controller for Mobile Robot Navigation. IEEE International Conference on Fuzzy Systems. 2005. p. 319-324.

76. Baturone I, Gersnoviez A, Barriga A. Neuro-Fuzzy Techniques to Optimize an FPGA Embedded Controller for Robot Navigation. ELSEVIER Applied Soft Computing. 2014;21:95-106.

77. Ma X, Li X, Qiao H. Fuzzy Neural Network-Based Real-Time SelfReaction of Mobile Robot in Unknown Environments. ELSEVIER Mechatronic. 2001;11(8):1039-1052.

78. Imen M, Mohammad M, Shoorehdeli MA. Tracking Control of Mobile Robot Using ANFIS. IEEE International Conference on Mechatronics and Automation. 2011. p. 422-427.

79. Ganapathy V, Yun SC, Ng J. Fuzzy and Neural Controllers for Acute Obstacle Avoidance in Mobile Robot Navigation. IEEE/ASME International Conference on Advanced Intelligent Mechatronics. 2009. p. $1236-1241$.

80. Zhao T, Wang YA. Neural-Network based Autonomous Navigation System using Mobile Robots. IEEE 12th International Conference on Control, Automation, Robotics \& Vision (ICARCV). 2012. p. 1101-1106.

81. Kumar D, Dhama K. Neuro-Fuzzy Control of an Intelligent Mobile Robot. IEEE Second International Conference on Advanced Computing \& Communication Technologies (ACCT). 2012. p. 106-111.

82. Song KT, Sheen LH. Heuristic Fuzzy-Neuro Network and its Application to Reactive Navigation of a Mobile Robot. ELSEVIER Fuzzy Sets and Systems. 2000;110(3):331-340.

83. Lee CH, Chiu MH. Recurrent Neuro Fuzzy Control Design for Tracking of Mobile Robots via Hybrid Algorithm. ELSEVIER Expert Systems with Applications. 2009;36(5):8993-8999.

84. Deshpande SU, Bhosale SS. Adaptive Neuro-Fuzzy Inference System based Robotic Navigation. IEEE International Conference on Computational Intelligence and Computing Research (ICCIC). 2013. p. $1-4$

85. Rusu P, Petriu EM, Whalen TE, et al. Behavior-Based Neuro-Fuzzy Controller for Mobile Robot Navigation. IEEE Transactions on Instrumentation and Measurement. 2003;52(4):1335-1340.

86. Pothal JK, Parhi DR. Navigation of Multiple Mobile Robots in a Highly Clutter Terrains using Adaptive Neuro-Fuzzy Inference System. ELSEVIER Robotics and Autonomous Systems. 2015;72:48-58.

87. Ng KC and Trivedi MM. A Neuro-Fuzzy Controller for Mobile Robot 
Navigation and Multirobot Convoying. IEEE Transactions on Systems, Man, and Cybernetics. 1998;28(6):829-840.

88. Demirli K, Khoshnejad M. Autonomous Parallel Parking of a Car-Like Mobile Robot by a Neuro-Fuzzy Sensor-Based Controller. ELSEVIER Fuzzy Sets and Systems. 2009;160(19):2876-2891.

89. Al-Mayyahi A, Wang W, Birch P. Adaptive Neuro-Fuzzy Technique for Autonomous Ground Vehicle Navigation. Robotics. 2014;3(4):349-370.

90. Pradhan SK, Parhi DR, Panda AK. Neuro-Fuzzy Technique for Navigation of Multiple Mobile Robots. SPRINGER Fuzzy Optimization and Decision Making. 2006;5(3):255-288.

91. Algabri M, Mathkour H, Ramdane H. Mobile Robot Navigation and Obstacle-Avoidance Using ANFIS in Unknown Environment. International Journal of Computer Applications. 2014;91(14):36-41.

92. Elshamli A, Abdullah HA, Areibi S. Genetic Algorithm for Dynamic Path Planning. IEEE International Conference on Electrical and Computer Engineering. 2004. p. 677-680.

93. Mohanta JC, Parhi DR, Patel SK. Path Planning Strategy for Autonomous Mobile Robot Navigation Using Petri-GA Optimisation. Computers and Electrical Engineering. 2011;37(6):1058-1070.

94. Kubota N, Morioka T, Kojima F, Fukuda T. Learning of Mobile Robots Using Perception-Based Genetic Algorithm. ELSEVIER Measurement. 2001;29(3):237-248.

95. Tuncer A, Yildirim M. Dynamic Path Planning of Mobile Robots with Improved Genetic Algorithm. ELSEVIER Computers and Electrical Engineering. 2012;38(6):1564-1572.

96. Ming L, Zailin G, Shuzi Y (1996). Mobile Robot Fuzzy Control Optimization Using Genetic Algorithm. ELSEVIER Artificial Intelligence in Engineering. 1996;10(4):293-298.

97. Hu Y, Yang SX, Xu LZ, et al. A Knowledge based Genetic Algorithm for Path Planning in Unstructured Mobile Robot Environments. IEEE International Conference on Robotics and Biomimetics (ROBIO). 2004. p. 767-772.

98. Liu Q, Lu YG, Xie CX. Fuzzy Obstacle-avoiding Controller of Autonomous Mobile Robot Optimized by Genetic Algorithm under Multi-obstacles Environment. IEEE 6th World Congress on Intelligent Control and Automation. 2006. p. 3255-3259.

99. Li Q, Zhang W, Yin Y, et al. An Improved Genetic Algorithm of Optimum Path Planning for Mobile Robots. IEEE 6th International Conference on Intelligent Systems Design and Applications (ISDA). 2006. p. 637-642.

100. Qu H, Xing K, Alexander T. An Improved Genetic Algorithm with CoEvolutionary Strategy for Global Path Planning of Multiple Mobile Robots. ELSEVIER Neurocomputing. 2013;120:509-517.

101. Algabri M, Mathkour H, Hedjar R, et al. Self-learning Mobile Robot Navigation in Unknown Environment Using Evolutionary Learning. Journal of Universal Computer Science. 2014;20(10):1459-1468.

102. Castillo O, Trujillo L, Melin P. Multiple Objective Genetic Algorithms for Path-Planning Optimization in Autonomous Mobile Robots. SPRINGER Soft Computing. 2007;11(3):269-279.

103. Arora T, Gigras Y, Arora V. Robotic Path Planning using Genetic Algorithm in Dynamic Environment. International Journal of Computer Application. 2014;89(11):8-12.

104. Metropolis N, Rosenbluth AW, Rosenbluth MN, et al. Equation of State Calculations by Fast Computing Machines. The Journal of Chemical Physics. 1953;21(6):1087-1092.

105. Yanar TA, Akyurek Z. Fuzzy Model Tuning Using Simulated Annealing. Expert Systems with Applications. 2011;38(7):8159-8169.

106. Chang CC, Song KT. Sensor-Based Motion Planning of a Mobile Robot in a Dynamic Environment. IEEE International Conference on Industrial Electronics, Control, Instrumentation. 1996. p. 766-771.

107. Martinez-Alfaro H, Gomez-Garcia S. Mobile Robot Path Planning and Tracking Using Simulated Annealing and Fuzzy Logic Control. ELSEVIER Expert Systems with Applications. 1998;15(3):421-429.

108. Zhu Q, Yan Y, Xing Z. Robot Path Planning Based on Artificial Potential Field Approach with Simulated Annealing. IEEE Proceedings of the Sixth International Conference on Intelligent Systems Design and Applications (ISDA). 2006. p. 622-627.

109. Precup RE, Radac MB, Dragos CA, et al. Simulated Annealing Approach to Fuzzy Modeling of Servo Systems. IEEE International Conference on Cybernetics. 2013. p. 267-272.

110. Janabi-Sharifi F, Vinke D. Integration of the Artificial Potential Field Approach with Simulated Annealing for Robot Path Planning. IEEE International Symposium on Intelligent Control. 1993. p. 536-541.

111. Tavares RS, Martins TC, Tsuzuki MDSG. Simulated Annealing with Adaptive Neighbourhood: A Case Study in Off-Line Robot Path Planning. Expert Systems with Applications. 2011;38(4):2951-2965.

112. Liang Y, Xu L. Global Path Planning for Mobile Robot based Genetic Algorithm and Modified Simulated Annealing Algorithm. In ACM Proceedings of Summit on Genetic and Evolutionary Computation. 2009. p. 303-308.

113. Nakamura E, Kehtarnavaz N. Optimization of Fuzzy Membership Function Parameters. IEEE International Conference on Systems, Man and Cybernetics. 1995. p. 1-6.

114. Hussein A, Mostafa H, Badrel-din M, Sultan O, Khamis A (2012) Metaheuristic Optimization Approach to Mobile Robot Path Planning. IEEE International Conference on Engineering and Technology (ICET). 2012. p. 1-6.

115. Miao H, Tian YC. Robot Path Planning in Dynamic Environments using a Simulated Annealing Based Approach. IEEE International Conference on Control, Automation, Robotics and Vision (ICARCV). 2008. p. 12531258.

116. Zhang Q, Ma J, Liu Q. Path Planning based Quad tree Representation for Mobile Robot Using Hybrid-Simulated Annealing and Ant Colony Optimization Algorithm. IEEE International World Congress on Intelligent Control and Automation. 2012. p. 2537-2542.

117. Gao M, Tian J. Path Planning for Mobile Robot Based on Improved Simulated Annealing Artificial Neural Network. IEEE Third International Conference on Natural Computation. 2007. p. 8-12.

118. Synodinos A, Aspragathos NA. Robot Path Planning of a Mobile Robot using Solid Modeling Techniques on Potential Fields. IEEE International Conference on Mechatronics and Embedded Systems and Applications (MESA). 2010. p. 549-553.

119. Zhao Y, Zu W. Real-Time Obstacle Avoidance Method for Mobile Robots Based on a Modified Particle Swarm Optimization. IEEE International Joint Conference on Computational Sciences and Optimization (CSO). 2009. p. 269-272.

120. Castillo O, Martinez-Marroquin R, Melin P, Valdez F, et al. Comparative Study of Bio-Inspired Algorithms Applied to the Optimization of Type1 and Type-2 Fuzzy Controllers for an Autonomous Mobile Robot. ELSEVIER Information sciences. 2012;192:19-38.

121. Zhang Y, Gong DW, Zhang JH. Robot Path Planning in Uncertain Environment using Multi-Objective Particle Swarm Optimization. ELSEVIER Neurocomputing. 2013;103:172-185.

122. Zhang Q, Li S. A Global Path Planning Approach based on Particle Swarm Optimization for a Mobile Robot. International Conference on Robotics, Control \& Manufacturing Technology World Scientific and Engineering Academy and Society (WSEAS). 2007. p. 263-267. 
123. Raja P, Pugazhenthi S. Path Planning for Mobile Robots in Dynamic Environments using Particle Swarm Optimization. IEEE International Conference on Advances in Recent Technologies in Communication and Computing (ARTCom). 2009. p. 401-405.

124. Masehian E, Sedighizadeh D. A Multi-Objective PSO-based Algorithm for Robot Path Planning. IEEE International Conference on Industrial Technology (ICIT). 2010. p. 465-470.

125. Wong C, Wang H, Li S. PSO-based Motion Fuzzy Controller Design for Mobile Robots. International Journal of Fuzzy Systems. 2008;10(1):284 292.

126. Li Q, Tang Y, Wang L, et al. A Specialized Particle Swarm Optimization for Global Path Planning of Mobile Robots. IEEE Third International Workshop on Advanced Computational Intelligence (IWACI). 2010. p. 271-276.

127. Huang HC. FPGA-Based Parallel Metaheuristic PSO Algorithm and its Application to Global Path Planning for Autonomous Robot Navigation. Journal of Intelligent \& Robotic Systems. 2014;76(3-4):475-488.

128. Chung HY, Hou CC, Liu SC. Automatic Navigation of a Wheeled Mobile Robot using Particle Swarm Optimization and Fuzzy Control. IEEE International Symposium on Industrial Electronics (ISIE). 2013. p. 1-6.

129. Shiltagh NA, Jalal LD. Optimal Path Planning for Intelligent Mobile Robot Navigation using Modified Particle Swarm Optimization. International Journal of Engineering and Advanced Technology. 2013;2(4):260-267.

130. Chatterjee A, Matsuno F. A Geese PSO Tuned Fuzzy Supervisor for EKF based Solutions of Simultaneous Localization and Mapping (SLAM) Problems in Mobile Robots. Expert Systems with Application. 2010;37(8):5542-5548.

131. Juang CF, Chang YC. Evolutionary-Group-based Particle-Swarm Optimized Fuzzy Controller with Application to Mobile-Robot Navigation in Unknown Environments. IEEE Transactions on Fuzzy Systems. 2011;19(2):379-39.

132. Lu L, Gong D. Robot Path Planning in Unknown Environments using Particle Swarm Optimization. IEEE International Conference on Natural Computation (ICNC). 2008. p. 422-426.

133. Allawi ZT, Abdalla TYA. PSO-Optimized Type-2 Fuzzy Logic Controller for Navigation of Multiple Mobile Robots. IEEE International Conference on Methods and Models in Automation and Robotics (MMAR). 2014. p. 33-39.

134. Dorigo M, Caro GD. Ant Colony Optimization: A New Meta-Heuristic. IEEE International Congress on Evolutionary Computation. 1999. p. $1470-1477$.

135. Guan-Zheng TAN, Huan H, Sloman A. Ant Colony System Algorithm for Real-Time Globally Optimal Path Planning of Mobile Robots. Acta Automatica Sinica. 2007;33(3):279-285.

136. Purian FK, Sadeghian E. Mobile Robots Path Planning using Ant Colony Optimization and Fuzzy Logic Algorithms in Unknown Dynamic Environments. IEEE International Conference on Control, Automation, Robotics and Embedded Systems (CARE). 2013. p. 1-6.

137. Bi Z, Yimin Y, Yisan X. Mobile Robot Navigation in Unknown Dynamic Environment Based on Ant Colony Algorithm. In IEEE WRI Global Congress on Intelligent Systems. 2009. p. 98-102.

138. Dong J, Liu B, Peng K, et al. Robot Obstacle Avoidance based on an Improved Ant Colony Algorithm. IEEE WRI Global Congress on Intelligent Systems. 2009. p. 103-106.
139. Ganapathy V, Jie TTJ, Parasuraman S. Improved Ant Colony Optimization for Robot Navigation. IEEE International Symposium on Mechatronics and its Applications (ISMA). 2010. p. 1-6.

140. Fan X, Luo X, Yi S, et al. Optimal Path Planning for Mobile Robots Based on Intensified Ant Colony Optimization Algorithm. IEEE International Conference on Robotics, Intelligent Systems and Signal Processing. 2003. p. 131-136.

141. Sariff NB, Buniyamin. Comparative Study of Genetic Algorithm and Ant Colony Optimization Algorithm Performances for Robot Path Planning in Global Static Environments of Different Complexities. IEEE International Symposium on Computational Intelligence in Robotics and Automation (CIRA). 2009. p. 132-137.

142. Hsu CC, Hou RY, Wang WY. Path Planning for Mobile Robots Based on Improved Ant Colony Optimization. IEEE International Conference on Systems, Man, and Cybernetics (SMC). 2013. p. 2777-2782.

143. Ganganath N, Cheng CT, Tse CK. An ACO-based Off-line Path Planner for Nonholonomic Mobile Robots. IEEE International Symposium on Circuits and Systems (ISCAS). 2014. p. 1038-1041.

144. Juang CF, Hsu CH. Reinforcement Ant Optimized Fuzzy Controller for Mobile-Robot Wall-Following Control. IEEE Transactions on Industrial Electronics. 2009;56(10):3931-3940.

145. Hsu CH, Juang CF. Evolutionary Robot Wall-Following Control using Type-2 Fuzzy Controller with Species-DE-Activated Continuous ACO. IEEE Transactions on Fuzzy Systems. 2013;21(1):100-112.

146. Juang CF, Lai MG, Zeng WT. Evolutionary Fuzzy Control and Navigation for Two Wheeled Robots Cooperatively Carrying an Object in Unknown Environments. IEEE Transactions on Cybernetics. 2015;45(9):17311743.

147. Hsu CH, Juang CF. Multi-Objective Continuous-Ant-ColonyOptimized FC for Robot Wall-Following Control. IEEE Computational Intelligence Magazine. 2013;8(3):28-40.

148. Chen X, Kong Y, Fang X, et al. A Fast Two-Stage ACO Algorithm for Robotic Path Planning. Neural Computing and Applications. 2013;22(2):313-319.

149. Hossain MA, Ferdousand I. Autonomous Robot Path Planning in Dynamic Environment using a New Optimization Technique Inspired by Bacterial Foraging Technique. Robotics and Autonomous Systems. 2015;64:137-141.

150. Liang XD, Li LY, Wu JG, et al. Mobile Robot Path Planning based on Adaptive Bacterial Foraging Algorithm. Journal of Central South University. 2013;20(12):3391-3400.

151. Brand $\mathrm{M}, \mathrm{Yu}$ XH.Autonomous Robot Path Optimization using Firefly Algorithm. IEEE International Conference on Machine Learning and Cybernetics (ICMLC). 2013.p. 1028-1032.

152. Mohajer B, Kiani K, Samiei E, et al. A New Online Random Particles Optimization Algorithm for Mobile Robot Path Planning in Dynamic Environments. Hindawi Mathematical Problems in Engineering. 2013. p. 1-9.

153. Luo RC, Chang CC. Multisensor Fusion and Integration: A Review on Approaches and Its Applications in Mechatronics. IEEE Transactions on Industrial Informatics. 2012;8(1):49-60. 